\title{
The photometry of hollow light guides
}

\author{
A Rosemann ${ }^{a}$ PhD, S-H Kloss ${ }^{b}$ PhD, T Müller $^{c}$ PhD, S Aydinli ${ }^{a}$ PhD and $\mathbf{H}$ Kaase ${ }^{a}$ \\ a Technical University of Berlin, Fachgebiet Lichttechnik, Berlin, Germany \\ ${ }^{\mathrm{b}}$ KMT Consult beratende Ingenieure, Dr. Kloss \& Partner GmbH, Bielefeld, Germany \\ c LichtVision $\mathrm{GmbH}$, Berlin, Germany
}

Received 28 July 2003; Revised 9 January 2004; accepted 27 April 2004

\begin{abstract}
Light-pipes described in this paper guide light along an axis allowing it to escape from its surface for illumination purposes. Light is coupled into them by a beamer luminaire. The performance of a hollow light guide very much depends on the quality of the materials used to guide the light. The luminous characteristics of these materials influence a light-pipe's overall performance. The first section of this paper describes measurement techniques for light incidence resolved measurements on materials. Common co-ordinate systems for describing the geometry and a data format to store the data are shown. The second section of this paper deals with light-pipe goniophotometry. A goniophotometer for lightpipes has been constructed at the Technical University of Berlin. To evaluate the results measurements are compared to these from a real installation.
\end{abstract}

\section{List of symbols}

$\theta$

angle to describe light incidence and observer direction regarding the IEA TASK 21 co-ordinate system

$\varphi$ angle to describe light incidence and observer direction regarding the IEA TASK 21 co-ordinate system

$\alpha$ angle to describe light incidence and observer direction regarding the EUMELDAT co-ordinate system

$\gamma \quad$ angle to describe light incidence and observer direction regarding the EUMELDAT co-ordinate system

$\tau_{\mathrm{D} 65}$ luminous transmittance for standard illuminant D65

$\tau_{\text {dif }}$ luminous transmittance for diffuse light incidence

Address for correspondence: A Rosemann, Technical University of Berlin, Fachgebiet Lichttechnik, Sekr. E6, Einsteinufer 19, 10587 Berlin, Germany. E-mail: Lichttechnik@ee.tu-berlin.de
$L$

E illuminance

I luminous intensity

$r \quad$ distance

$\varepsilon_{2} \quad$ angle describing the light incidence on a photocell

$\eta_{S, i} \quad$ luminous efficacy of light-pipe segment $i$

$\Phi_{i} \quad$ luminous flux escaping from light-pipe segment $i$

$\Phi_{0} \quad$ total luminous flux entering a light-pipe

$\eta_{\text {pipe }} \quad$ luminous efficacy of a light-pipe

$\eta_{L B} \quad$ luminous efficacy of a light-pipe considering the beamer luminaire

$\eta_{L B, S O}$ luminous efficacy of the beamer luminaire

$e_{\text {mean }}$

$e_{\max }$

$q_{m}$

$q_{\mathrm{rel}}$ calculation error for mean illuminance maximum calculation error for an illuminance distribution

RMS error for the illuminance differences relative RMS error 


\section{Measurement on components}

The history of hollow light guides shows that the main improvements in performance have always followed an advance in the technology of materials. ${ }^{1}$ Good material characteristics with special regard to their lighting quantities are therefore an important basis for efficient hollow light guides. One material that has been the focus of many light-pipe developments is prismatic film. It allows light to undergo total internal reflection if the light reaching the film's surface meets the appropriate angular criteria.

\subsection{Measuring luminous quantities depending on the light incidence and observer direction}

Since the light reaching the prismatic film only undergoes total internal reflection if the Snell's angle criterion determined by Snell's law is met, the luminous transmittance and the luminous reflectance of the film depend on the light incidence. Measurements on the luminous transmittance of the prismatic film have to take this aspect into consideration.

\subsection{Co-ordinate systems}

The co-ordinate system for describing the incident light can be the one defined by the IEA (International Energy Agency) TASK 21 in the Source Book Daylight in Buildings ${ }^{2}$ (shown in Figure 1).

$\theta$ is the angle between the (positive orientated) $z$-axis and $\varphi$ is the angle to the reference plane defined by the $x$ - and $z$-axis.

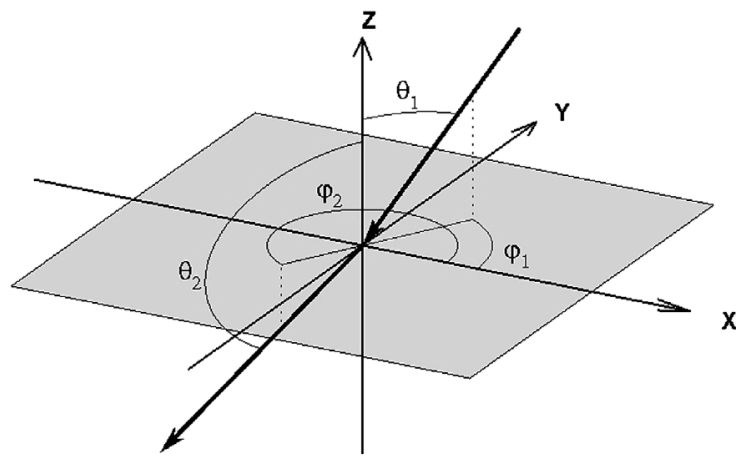

Figure 1 IEA co-ordinate system to define the light incidence $^{3}$

In most cases, this co-ordinate system is not very suitable for practical applications which take the sun's position into account. Most daylighting systems are vertically placed in the façade. Therefore, a new co-ordinate system was proposed by Herrmann et al. (see Figure 2). ${ }^{3}$ This new co-ordinate system allows the description of the light incidence and observer direction by an azimuth $(\alpha)$ and elevation angle $(\gamma)$. This approach is more practical.

For both co-ordinate systems, the subscripts 1 and 2 indicate light incidence and observer direction respectively. To transform IEA-coordinates (described by the angles $\theta$ and $\varphi$ ) into $\gamma, \alpha$-co-ordinates, the following equations are used (for either subscript):

$$
\gamma=\arcsin (\sin \theta \cdot \sin \varphi)
$$

For the transformation from $\gamma, \alpha$-co-ordinates into the IEA-co-ordinates, the following equations apply:

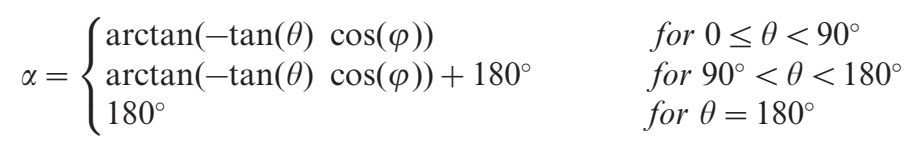




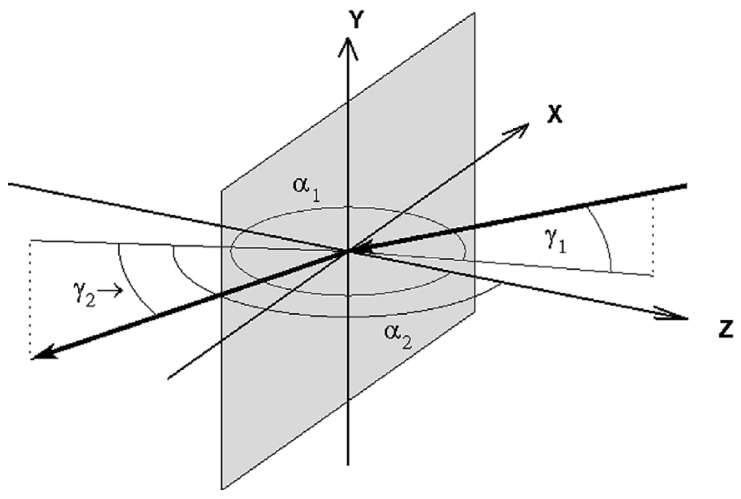

Figure 2 EUMELDAT co-ordinate system for describing the light incidence and observer direction ${ }^{3}$

$$
\theta=\arccos (\cos (\gamma) \cos (\alpha))
$$

$$
\varphi=\left\{\begin{array}{c}
90^{\circ} \\
\arctan \left(\frac{-\tan (\gamma)}{\sin (\alpha)}\right)+180^{\circ} \\
270^{\circ} \\
\arctan \left(\frac{-\tan (\gamma)}{\sin (\alpha)}\right)
\end{array}\right.
$$

\subsection{Luminous transmittance $\tau_{\mathrm{D} 65}$}

Luminous transmittance $\tau_{\mathrm{D} 65}$ for the standard illuminant D65 for different light incidences $\left(\gamma_{1}, \alpha_{1}\right)$ can be obtained by two basic methods:

- integrating Ulbricht sphere

- goniophotometer

The light entering the integrating sphere produces an indirect illuminance on the sphere's inner surface which is proportional to the luminous flux transmitted by the sample. Errors occur for photometric reasons (e.g., $V(\lambda)$-match of the photoelectric cell, diameter of the sphere, size of the sphere's opening, illuminated area of the sample). The position of the photoelectric cell and the inner surface's reflectance characteristics do also have an influence of the measured data. The inner surface's reflectance may vary slightly hence the photocurrent depends on the position of the photoelectric cell.

The luminous transmittance for diffuse light incidence can be calculated from the following equation:

$$
\begin{aligned}
\tau_{d i f}= & \frac{1}{\pi} \int_{\alpha_{1}=-\pi / 2}^{\pi / 2} \int_{\gamma_{1}=-\pi / 2}^{\pi / 2} \tau\left(\gamma_{1}, \alpha_{1}\right) \cdot \cos ^{2}\left(\gamma_{1}\right) \\
& \cdot \cos \left(\alpha_{1}\right) \cdot d \gamma_{1} \cdot d \alpha_{1}
\end{aligned}
$$

for $\alpha=0,180^{\circ}, 360^{\circ}$ and $\gamma>0$

for $0<\alpha<180^{\circ}$

for $\alpha=0,180^{\circ}, 360^{\circ}$ and $\gamma<0$

for $180^{\circ}<\alpha<360^{\circ}$

With an integrating sphere the luminous reflectance for a range of angles of incidence can also be measured. The azimuth angle $\alpha_{1}$ can have discrete values $\left(8^{\circ}, 20^{\circ}-70^{\circ}\right.$ in steps of $10^{\circ}$ ). Azimuth angles greater than $70^{\circ}$ illuminate a very large area of the surface so the error of the measurement data may become unacceptable.

A goniophotometer measures data which take into account the direction of the observer for transmitted light. The transmission can be computed for a range of direction angles from the equation: 


$$
\begin{aligned}
\tau\left(\gamma_{1}, \alpha_{1}\right)= & \int_{\alpha_{2}=\pi / 2}^{3 \pi / 2} \int_{\gamma_{2}=-\pi / 2}^{\pi / 2} q\left(\gamma_{1}, \alpha_{1}, \gamma_{2}, \alpha_{2}\right) \cdot \cos ^{2}\left(\gamma_{2}\right) \\
& \cdot \cos \left(\alpha_{2}\right) \cdot d \gamma_{2} \cdot d \alpha_{2}
\end{aligned}
$$

with

$$
q\left(\gamma_{1}, \alpha_{1}, \gamma_{2}, \alpha_{2}\right)=\frac{L\left(\gamma_{1}, \alpha_{1}, \gamma_{2}, \alpha_{2}\right)}{E}
$$

being the spatial distribution of the luminance coefficient. A 3D-graphical presentation of the data as shown in Figure 3 helps to demonstrate the optical behaviour of the prismatic film. The luminous transmittance for different light incidents is represented by different colours in the plot. The black area represents the light incidences that will undergo total internal reflection.

\subsection{Bi-directional measurements}

This section describes the measurements of the BDTF (Bi-directional Transmittance $D$ istribution $F$ unction) or BDRF (Bi-directional Reflectance $D$ istribution Function) i.e., the spatial distribution of the luminance coefficient $q$ depending on the angles of the light incidence and the observer direction.

Bi-directional measurements are carried out with goniophotometers. The main sources of error include the size of the sample surface's structure and the size of the illuminated area. The coarseness of the structure of the sample in relation to the illuminated area may cause these errors.

Table 1 shows appropriate light incidences in the $\gamma, \alpha$ co-ordinate-system for bi-directional measurements. These values are the rounded values for subdividing the hemisphere for
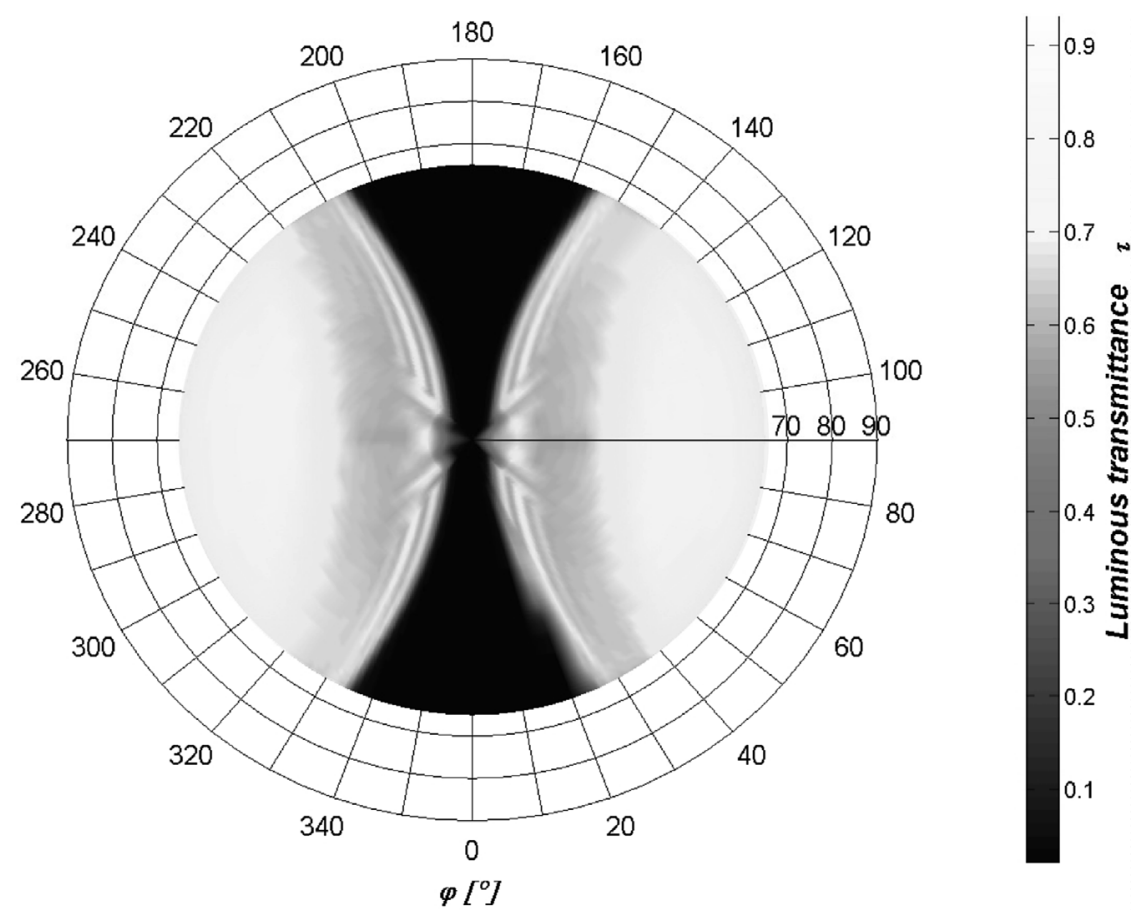

Figure 3 Luminous transmittance of the prismatic film for different light incidences 
Table 1 Light incidences for bi-directional measurements in the $\gamma, \alpha$-co-ordinate, system

\begin{tabular}{rrl}
\hline$\gamma_{1}$ in & $\begin{array}{l}\alpha_{1} \text {-step } \\
\text { in }{ }^{\circ}\end{array}$ & $\alpha_{1}$ in \\
\hline 0 & 12 & $0,12,24,36,48,60,72,84$ \\
12 & 12 & $0,12,24,36,48,60,72,84$ \\
24 & 15 & $0,15,30,45,60,75,90$ \\
36 & 15 & $0,15,30,45,60,75,90$ \\
48 & 18 & $0,18,36,54,72,90$ \\
60 & 24 & $0,24,48,72$ \\
72 & 40 & $0,40,80$ \\
84 & - & 0 \\
0 & -12 & $-12,-24,-36,-48,-60,-72,-84$ \\
12 & -12 & $-12,-24,-36,-48,-60,-72,-84$ \\
24 & -15 & $-15,-30,-45,-60,-75,-90$ \\
36 & -15 & $-15,-30,-45,-60,-75,-90$ \\
48 & -18 & $-18,-36,-54,-72,-90$ \\
60 & -24 & $-24,-48,-72$ \\
72 & -40 & $-40,-80$ \\
-12 & 12 & $0,12,24,36,48,60,72,84$ \\
-24 & 15 & $0,15,30,45,60,75,90$ \\
-36 & 15 & $0,15,30,45,60,75,90$ \\
-48 & 18 & $0,18,36,54,72,90$ \\
-60 & 24 & $0,24,48,72$ \\
-72 & 40 & $0,40,80$ \\
-84 & - & 0 \\
-12 & -12 & $-12,-24,-36,-48,-60,-72,-84$ \\
-24 & -15 & $-15,-30,-45,-60,-75,-90$ \\
-36 & -15 & $-15,-30,-45,-60,-75,-90$ \\
-48 & -18 & $-18,-36,-54,-72,-90$ \\
-60 & -24 & $-24,-48,-72$ \\
72 & -40 & $-40,-80$ \\
\hline
\end{tabular}

luminance measurements proposed by Tregenza $^{4}$ transformed into the $\gamma, \alpha$ co-ordinate system.

The number of measurements can be decreased if the sample's surface shows certain symmetries e.g., if the structure of the sample is symmetrical about a plane through a normal to the surface sample's surface. Measurements need only to be taken on one side of the plane of symmetry.

\subsection{Data format for direction resolved quantities}

To store the data in a standardized way, a data format was developed at the Technical University of Berlin. The data format described here is not restricted to the luminous characteristics mentioned above but can also be used to store the energetic characteristics of daylighting systems (e.g., solar factor).

Similar to the European data format for the photometric description of luminaires, EULUMDAT, $^{5}$ a data format for describing the direction resolved quantities of materials and systems, is proposed in this paper. EUMELDAT is the abbreviation for European Material, Energetic and Luminous Data. This data format describes the characteristics of the quantities described above in a standardized way. For any material or system, it helps designers to use the data in computer simulation software packages for optimization with respect to luminous and energetic properties. The data on the prismatic film (shown in Figure 3) can be stored in a EUMELDAT-file. This data can be used to calculate the luminous properties of a hollow light guide, or any other luminaire using this material, by ray tracing.

The values are saved independently of the platform in an ASCII-file. The files can be edited by commonly available editors. The negative trade-off of this approach is a larger file size. This does not introduce a major disadvantage on modern computers.

For a material or daylight system there are different files. All EUMELDAT-files have the file extension '.mel'. The filename consists of information on:

- material name

- additional system parameters (e.g., tilt angle of lamellae, thickness of glazing etc.)

- abbreviation of the measured quantity (see Table 2)

- short name of the laboratory which carried out the measurements.

A EUMELDAT-file is divided into a header section containing descriptive information and a data section with the main data. In the header section, each line starts with the number sign '\#'. The end of a line is formed by the ASCII-codes for LF and CR. The language for the descriptive part of the file should be English. Characters that are 
Table 2 Terms for measurement quantities in EUMELDAT

\begin{tabular}{ll}
\hline Identifier & Quantity \\
\hline tau & $\tau_{\mathrm{D} 65}$ luminous transmittance \\
rho & $\rho_{\mathrm{D} 65}$ luminous reflectance \\
$\mathrm{g}$ & solar factor \\
$\mathrm{q}$ & $q$ luminance coefficient \\
taue & $\tau_{\mathrm{e}}$ radiant transmittance \\
qi & $q_{i}$ secondary heat transfer factor \\
alpha1 & $\alpha_{1}$ \\
gamma1 & $\gamma_{1}$ \\
\hline
\end{tabular}

not part of the English alphabet are not allowed (e.g., the German 'umlaut' such as ä, ö, ü etc.).

The decimal point is ' $\because$. Numerical values within one line are separated by the TABcharacter. The end of the file is indicated by the line '\#END'.

In the data section of the file there are triples of values for the light incidence, the observer direction and the measurement value. The first column contains the $\gamma$-values.

The main advantage of EUMELDAT is the fact that this data format allows storage of several quantities in one file. Since there had previously been no data format to store these different types of information, EUMELDAT was developed. The way EUMELDAT is defined is closely related to the EULUMDAT format, which describes the luminous intensity distributions of luminaires.

\section{Photometry of light-pipe systems}

At the present time there are no standards for the measurement of the luminous intensity distribution of hollow light guides (HLG). The reason for this lack is that the length of hollow light guides $(4 \mathrm{~m}-30 \mathrm{~m})$ makes it impossible to measure light-pipes with conventional photometric apparatus.

New quantities for describing the lighting characteristics are defined. They are related to common lighting quantities. For a photometric description, light-pipes are divided into several parts (segments), each of which is treated as a single luminaire while being measured. The quantities are segment related quantities.

\subsection{Measurement technique}

Owing to their length, hollow light guide systems cannot be measured easily. The total luminous flux leaving a luminaire is usually measured in integrating spheres or with goniophotometers that integrate the illuminance distribution around the luminaire. Light-pipes require rather large measurement devices for such measurements. The same problem occurs, if the luminous intensity distribution of these systems needs to be measured. The conventional photometric approach is based on the inverse square law:

$$
I=\frac{E \cdot r^{2}}{\cos \left(\varepsilon_{2}\right)}
$$

This law is only valid for point light sources, which do not exist in reality. Therefore the measurement distance has to be chosen in such a way that the error due to the size of the light emitting area is acceptable. As a rule of thumb, a distance of 10 times this area's maximum dimension is taken. This rule of thumb is not universally applicable. The measurement distance depends on the following quantities:

- size of the light emitting surface

- luminous intensity distribution of the luminaire

- luminance distribution of the light emitting area

- maximum acceptable measurement error size of the photocell.

Additionally, the measurement data cannot be used for photometric calculations in small rooms i.e., rooms that are so small that the inverse square law is not applicable. The distance between the light-pipe's centre and any surface of the room itself would 
be smaller than the measurement distance. Also, the luminance distribution along the light-pipe is not constant so that even more errors would be introduced into the calculation.

A possible approach is near-field photometry. Here, the inverse square law is not considered so that the distance between the photometer head and the measurement object does not have an influence on the results. Luminance is a quantity that does not change with distance. Near-field photometry is based on luminance distribution measurements. The luminous intensity distribution is computed from the luminance data. There are literature sources that describe near-field photometry. ${ }^{6-8}$

\subsection{New photometric quantities}

The EULUMDAT-files of a light-pipe can be used in lighting simulation tools and positioning them is easier if the segments into which the light-pipes are split are all of the same length. A light-pipe would then be treated as several single luminaries. Usually a length of about $1 \mathrm{~m}$ is chosen. Segments of this length can be measured by the goniophotometer. The smaller size of the light emitting surface allows calculations to be made with lighting software (see section 2.2.3 Evaluation of the measurement results).

The luminous intensity distribution and the luminous flux of each segment are measured directly. Other relevant quantities (e.g., glare class) can be computed using the measurement data.

\subsubsection{Definitions of relevant quantities}

Owing to the segmentation of hollow light guides, the conventional lighting quantities have to be modified. Basically, the quantities have to be split up into segmentation related ones. ${ }^{9}$

Segment luminous flux: The segment luminous flux $\Phi_{i}$ is the luminous flux that leaves the segment $i$.
Efficiencies: The segment efficiency $\eta_{S, i}$ is the ratio of the segment luminous flux and the initial luminous flux $\Phi_{0}$ :

$$
\eta_{S, i}=\frac{\Phi_{i}}{\Phi_{0}}
$$

Owing to the fact that all efficiencies are related to the initial luminous flux, the efficiency of the light-pipe $\eta_{\text {pipe }}$ can be calculated as follows:

$$
\eta_{\text {pipe }}=\sum_{i=1}^{N} \eta_{S, i}
$$

The overall efficiency of the system, taking the light source (SQ) into account, is:

$$
\eta_{L B}=\eta_{L B, S Q} \cdot \sum_{i=1}^{N} \eta_{S, i}
$$

$\eta_{L B, S Q}$ is the luminaire efficiency of the hollow light guide system.

Light output angle: The light output angle is the angle of a light-pipe's transparent area that allows light to escape. The corresponding light output angle of a light-pipe coupling out light from the lower half of the light-pipe only would be $180^{\circ}$. Typical light output angles of hollow light guide systems are $90^{\circ}, 120^{\circ}, 180^{\circ}$ and $360^{\circ}$.

\subsubsection{Goniophotometer for light-pipes}

As part of the European research project ARTHELIO, ${ }^{10,11}$ a goniophotometer for measuring the luminous flux and luminous intensity distribution of light-pipes was built up at the Technical University of Berlin (shown in Figures 4 and 5). It consists of two main parts. First, there is a rail system (S) mounted below the ceiling with an overall length of $63 \mathrm{~m}$ which is an aluminium extrusion. It is thus possible to measure hollow light guides with a length of up to $30 \mathrm{~m}$. The profile's cross-sectional area was optimized to give 


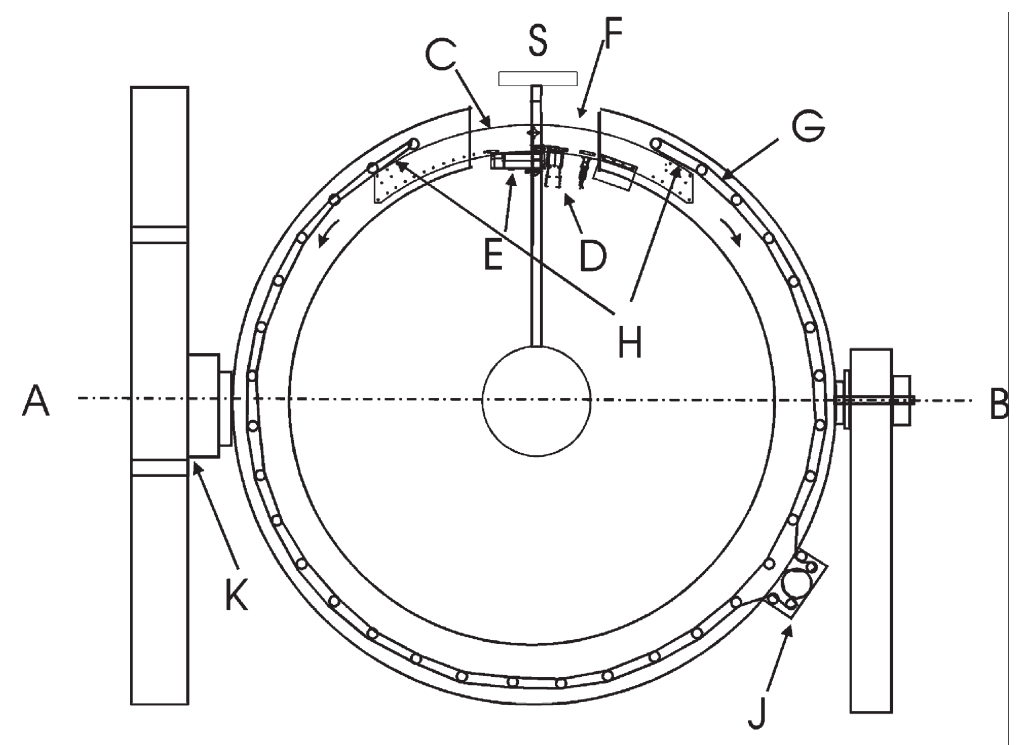

Figure 4 Diagram of the goniophotometer for light-pipes

high stability to the system. Along this rail is attached to each carriage. Its length can system there are numerous carriages attached be changed by a handle so that light-pipes to the rail system by rollers. These carriages of different diameters can be held in the axis are driven by a toothed belt. A telescopic arm of the system. The relative position of the

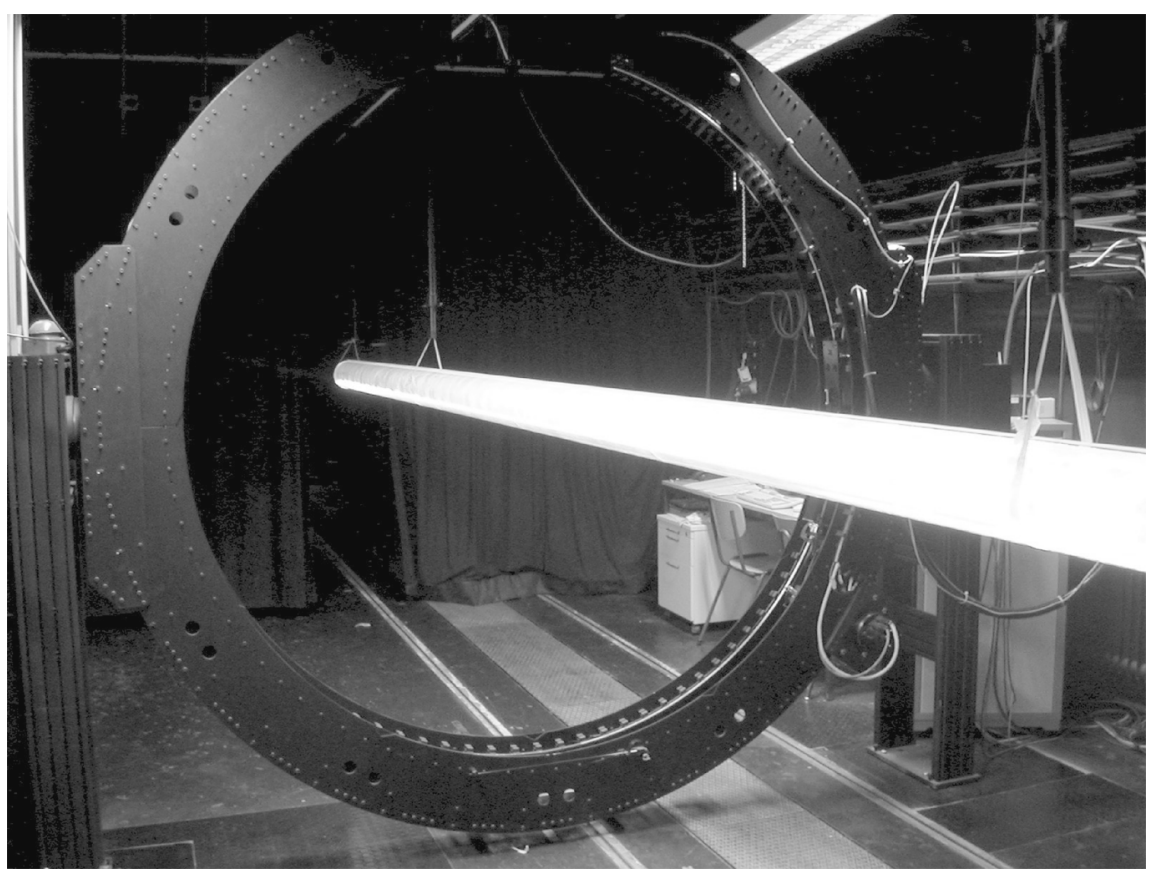

Figure 5 TUB-Goniophotometer for hollow light guides 
sample holders can be changed by a special mechanical device.

The second part is the goniophotometer itself, consisting of a circular arc concentric with the middle of the rail system. The lightpipes are driven along their horizontal axis. The arc is attached to two spindles and can be rotated around the horizontal axis $(\mathrm{A}-\mathrm{B})$. Within the arc there is a sensor carriage $(C)$, driven by a toothed belt $(\mathrm{G})$. This carriage is moved along rails $(\mathrm{H})$. The carriage has an angular size of $70^{\circ}$ along the arc. It moves four devices: the $\mathrm{CCD}$ camera (E) for relative luminance distribution measurements, a photocell (D) and a photocurrent amplifier for absolute illuminance measurements, and a laser for adjusting the system (see Figure 6). This offset between the positions of the CCD camera and the photocell is corrected by the software. The arc contains a hole at the top (F) so it can go all around a light-pipe while measuring the end part of the system. The carriage is able to move into this gap allowing it to make a complete revolution. It is driven by a motor unit (J). The wires for measurement and control signals enter the arc from one side $(\mathrm{K})$.

All these mechanical and photometric devices are controlled by a computer, which carries out the measurements, converts the near field data to far field data and finally saves the data in a file. With the goniophotometer it is possible to take luminous intensity measurements from all the solid angles apart from the ones that are covered by the measuring object itself. The missing data can be obtained by interpolation. ${ }^{12}$

The calibration of the luminous flux was carried out by using a calibrated standard source. The accuracy was better than $2.4 \%$ and after the correction of the parts shaded by the lamp holder better than $1.6 \%$. Owing

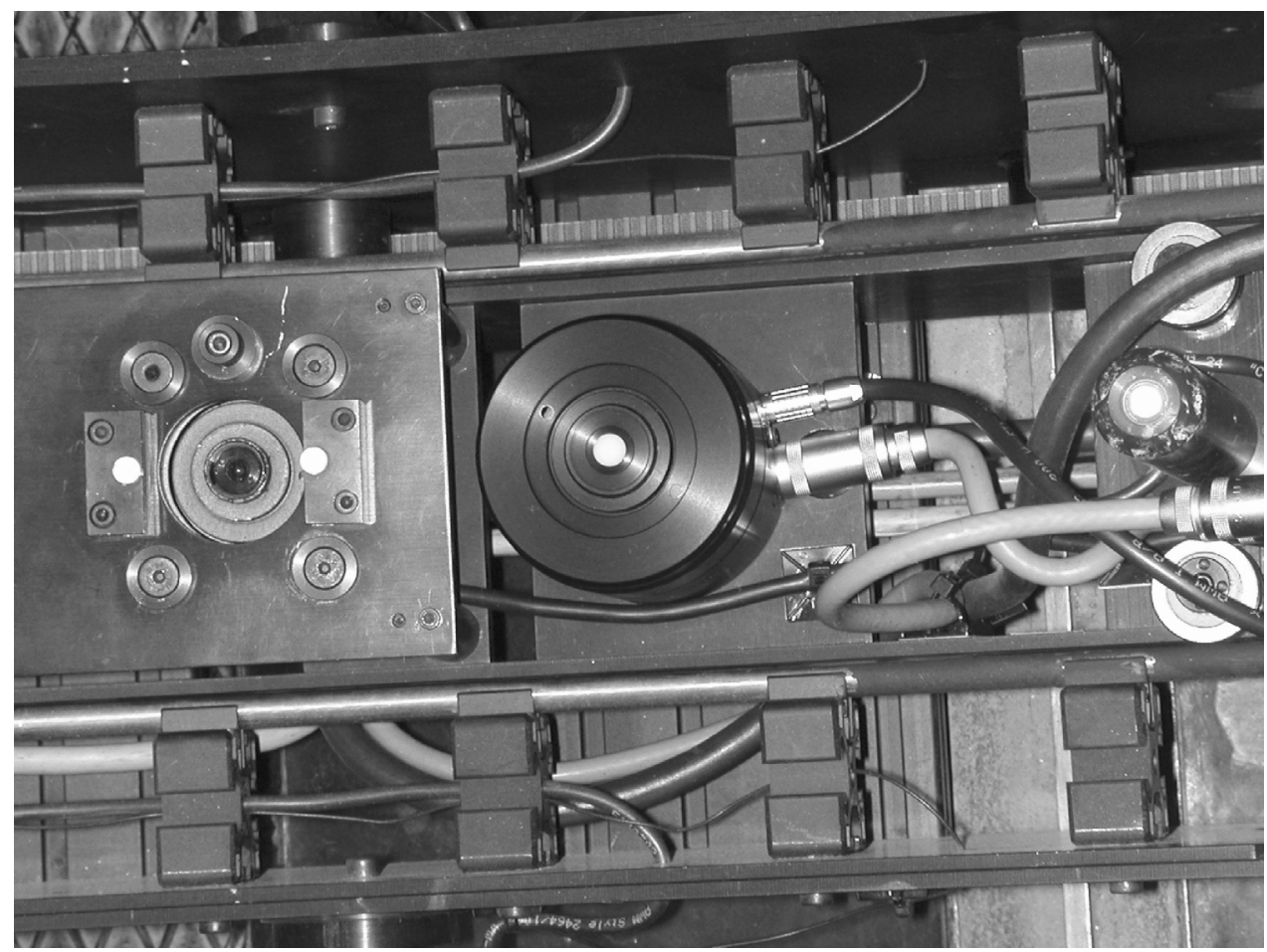

Figure 6 Sensor wagon with CCD-camera (left), photocell (centre) and laser (right) 
to the measurement technique used for the CCD camera, a direct calibration of the luminous intensity with a calibrated standard is not possible. Instead, a $2 \mathrm{~m}$ module with a standard fluorescent lamp inside and with an unmasked segment of $1 \mathrm{~m}$ was used to find the accuracy by comparing the results with that obtained from the moving mirror photometer. ${ }^{13}$ The relative error was less than $5 \% .{ }^{14}$

Examples for the luminous intensity distributions of two light-pipe systems are shown in Figures 7 and 8. Both systems are bidirectionally fed (i.e., with light injectors on both sides) and have a length of $6 \mathrm{~m}$. They differ in their light output angles which are $90^{\circ}$ (Figure 7 ) and $180^{\circ}$ (Figure 8 ).

\subsubsection{Evaluation of the measurement results}

To evaluate the measurement data collected by the goniophotometer for light-pipes, the data files containing the segment luminous intensity distributions were used for an illuminance calculation. The computation results for the goniophotometers are outlined in this section.

For comparison, measurements in a lightpipe installation in a windowless test room of the Technical University Berlin were compared with results of lighting calculations obtained from two different software packages. ${ }^{15}$ Both software packages were selected because they are freely available for download on the internet.

The room was $5.5^{\circ} \mathrm{m} \times 8.1^{\circ} \mathrm{m}$ with a height of $2.8 \mathrm{~m}$. The light-pipe had a diameter of

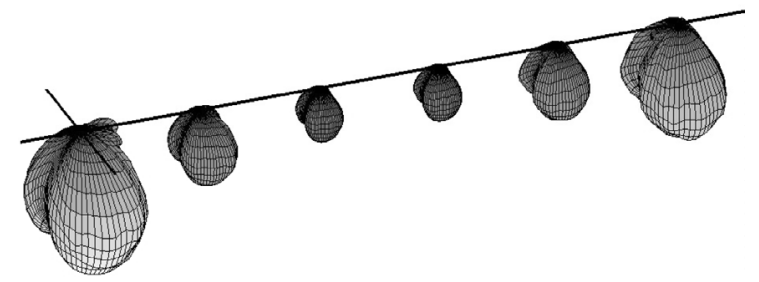

Figure 7 Luminous intensity distribution of a bi-directionally fed $6 \mathrm{~m}$ light-pipe with $90^{\circ}$ light output angle

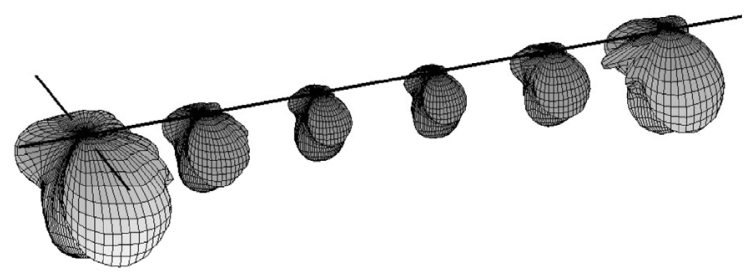

Figure 8 Luminous intensity distribution of a bi-directionally fed $6 \mathrm{~m}$ light-pipe with $180^{\circ}$ light output angle

$0.25 \mathrm{~m}$ and a length of $5 \mathrm{~m}$. It was illuminated by a sulphur lamp $(1.4 \mathrm{~kW})$. The test room is shown in Figure 9.

The illuminance distribution was measured and calculated on a $0.5 \mathrm{~m} \times 0.5 \mathrm{~m}$ grid, $0.85 \mathrm{~m}$ above the floor. The average measured illuminance was 1572 lux.

The comparison of the computed results and the measured data shows that the maximum difference occurs close to the walls. Therefore, the analysis compared the whole room as well as the room without a $0.5 \mathrm{~m}$ band close to the walls. The latter configuration will be called room without bands. The calculation error was computed for the mean illuminance $\left(e_{\text {mean }}\right)$ and for the maximum error in one reference point $\left(e_{\max }\right)$. Table 3 shows the comparison.

Owing to a very uneven illuminance distribution, the values are not very comparable so that the RMS value for the differences of the measured and computed data was calculated as well according to the following equation:

$$
q_{m}=\sqrt{\frac{1}{n} \sum_{i=1}^{n}\left(E_{\text {meas }, i}-E_{\text {calc }, i}\right)^{2}}
$$

For relative values, the RMS value was divided by the measured mean illuminance of the reference area:

$$
q_{\text {rel }}=\frac{q_{m}}{\bar{E}_{\text {meas }}}
$$

The results are given in Table 4. 


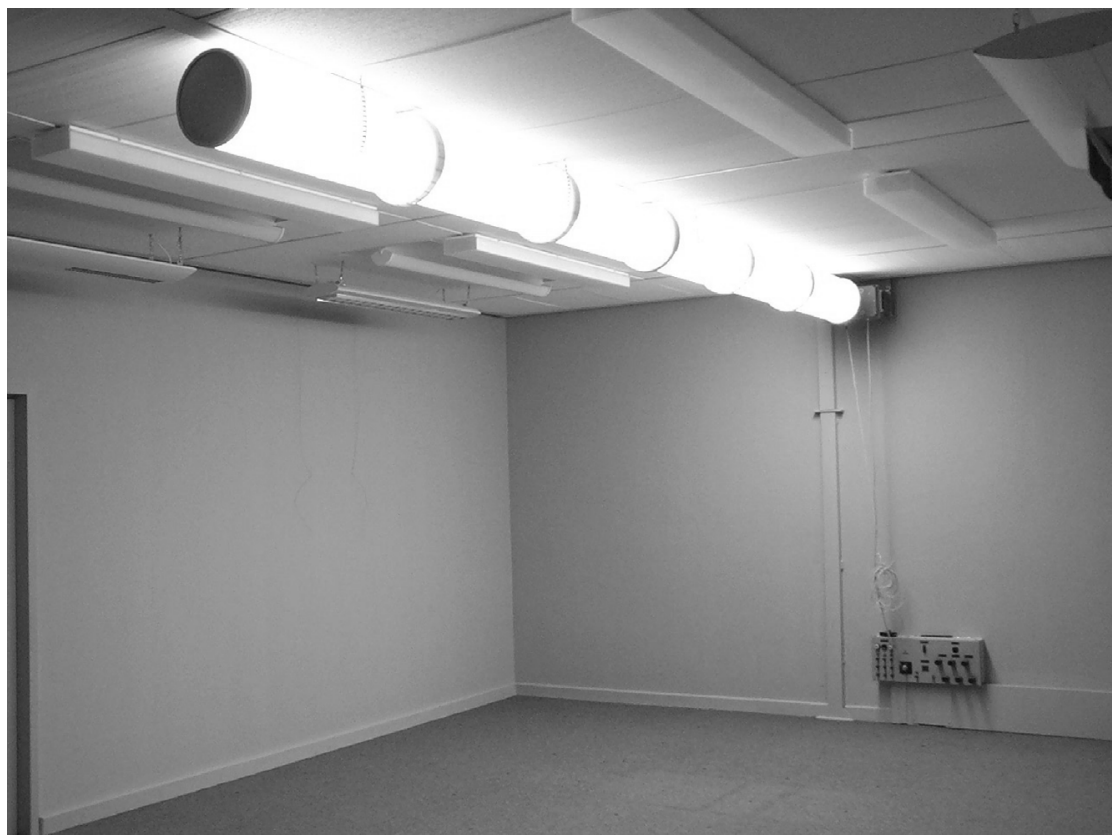

Figure 9 Light-pipe installation in a testroom

The tests show that the data obtained with the goniophotometer is suitable for simulations with the selected software packages. Within a band of $0.5 \mathrm{~m}$ close to the walls, the calculation results may not be very exact, which is due to the computation algorithm radiosity. The results are of higher quality if this band is not taken into account for illuminance calculations.

\section{Conclusion}

For improving the performance of luminaries, especially light-pipes, knowledge of the material characteristics are of major importance.
With a standardized data format, these materials can be described and used for the optimization processes involved in design. Hollow light guide systems can be simulated by ray tracing programs if the material characteristics (e.g., reflectance) are known for different light incidences. For the utilization of daylight in light-pipes equipped with any material, the luminous flux transported by a light-pipe can be calculated with respect to the luminance distribution of the sky.

With the goniophotometer for light-pipes the luminous intensity distribution of hollow light guide systems with an artificial light source can be obtained. The measurement

Table 3 Comparison of measured and calculated differences of a light-pipe installation

\begin{tabular}{lllll}
\hline & Whole room & \multicolumn{2}{l}{ Room without bands } & \\
\cline { 3 - 5 } & $e_{\text {mean }}$ in $\%$ & $e_{\text {max }}$ in $\%$ & $e_{\text {mean }}$ in $\%$ & $e_{\text {max }}$ in $\%$ \\
\hline Program 1 & 10 & 31 & 6 & 17 \\
Program 2 & 6 & 22 & 3 & 14 \\
\hline
\end{tabular}


Table 4 Comparison of measured and calculated differences of a light-pipe installation RMS-calculation

\begin{tabular}{|c|c|c|c|c|}
\hline & \multicolumn{2}{|c|}{ Whole room } & \multicolumn{2}{|c|}{ Room without bands } \\
\hline & $q_{m}$ in lux & $q_{r e l}$ in $\%$ & $q_{m}$ in lux & $q_{\text {rel }}$ in $\%$ \\
\hline Program 1 & 162 & 10 & 151 & 7 \\
\hline Program 2 & 124 & 8 & 85 & 4 \\
\hline
\end{tabular}

results can be used by lighting software packages and lead to reliable results. This is very helpful for lighting designers so that they can consider light-pipe applications in the design process.

For daylight entering a light-pipe, the calculation of the segment luminous intensity distribution presents problems since the outside conditions vary rather quickly. For different light incidences the luminous intensity distributions of each segment have to be measured. The overall luminous intensity distribution of each segment can then be computed from superposition of all light incidences weighted by the luminance distribution of the feeding source (e.g., the sun and the sky). In the project ARTHELIO it has been shown that the utilization of diffuse skylight fed into a long light-pipe system (approximately $12 \mathrm{~m}$ ) is not sufficient for interior lighting. Heliostat systems that redirect and concentrate the direct sunlight perform much better in this case. The relative luminous intensity distribution of the light fed into the light-pipe does not vary much over time (if the sun shines) and as a consequence the interior lighting situation does not either.

\section{Acknowledgements}

This work on light-pipe goniophotometry was carried out within the European research project ARTHELIO funded by the European Commission. The development of the data format is one result of the German joint research project called 'Tageslichtnutzung in Gebäuden' funded by the German Federal Ministry of Trade and Industry.

\section{References}

1 Aizenberg J. From the history of hollow light guides and their efficient applications. Light Engineering 2000; 8: 2, 35-48.

2 IEA SHCP TASK 21 Source Book 'Daylight in Buildings' 2002.

3 Herrmann B, Rosemann A, Aydinli S, Kaase H. 'EUMELDAT' Ein europäisches Datenformat für strahlungsphysikalische und lichttechnische Kennzahlen von Tageslichtsystemen; Tagungsband Neuntes Symposium Innovative Lichttechnik in Gebäuden (OTTI Technologie-Kolleg) 2003, Kloster Banz/Staffelstein; 2003.

4 Tregenza PR. Subdivision of the sky hemisphere for luminance measurements. Lighting Res. Technol. 1987; 19: 13-14.

5 Stockmar A. Eulumdat-Ein LeuchtenDatenformat für den europäischen Beleuchtungsplaner; Licht 90, Tagungsband der 9. Gemeinschaftstagung der lichttechnischen Gesellschaften in Rotterdam, 1990.

6 Ashdown I. Near-field photometry: a new approach. J. Illum. Eng. Soc. 1993; Winter: 163-80.

7 Ashdown I. Solving the near-field problem. Lighting Magazine 1992; 6: 38-40.

8 Whitehead L, Kan P, Lui K, Jacob S. Near-field photometry of prism light guide luminaires using a CCD camera. J. Illum. Eng. Soc., Summer 1999; Summer: 3-9.

9 Rosemann A. Hohllichtleiter für Tageslichtnutzung. PhD thesis TU Berlin; Pflaum Verlag GmbH \& Co. KG; 2002; ISBN 3-7905-0862-4.

10 Müller T, Kloss S-H, Rosemann A, Kaase H. ARTHELIO-Ein Europäisches Forschungsprojekt zur kombinierten Nutzung von Tageslicht und dem Kunstlicht der

Lighting Res. Technol. 37,1 (2005) pp. 60-72 
Schwefellampe in Gebäuden; Tagungsband OTTI-Fünftes Symposium Innovative Lichttechnik in Gebäuden, Kloster Banz, 1999; 34-40.

11 Rosemann A, Kaase H. Daylight for building interiors; Proceedings of the CIE/ARUP Symposium on Visual Environment at the Royal Society, London: 2002.

12 Kloss S-H, Kaase H, Müller T, Rosemann A. Goniophotometrie an Hohllichtleitern; Tagungsband Licht 2000, Goslar; 2000.
13 Lindemuth F, Zwick P. Zum Stand der Lichtmesstechnik. LICHT 2/88, 1988; 122-25.

14 Kloss S-H. Ein Goniophotometer zur Messung des Lichtstromes und der Lichtstärkeverteilung von hohlen Lichtleitern (A goniophotometer for measuring the luminous flux and the luminous intensity distributions of hollow light guides) PhD thesis. TU Berlin, 2001.

15 Rosemann A, Kiwull N, Kloss S-H, Müller T, Kaase $\mathrm{H}$. Innenraumbeleuchtung mit Hohllichtleitern; Tagungsband Licht 2000, Goslar; 2000. 